\title{
IMPLICIT RELATIONAL ASSESSMENT PROCEDURE AND BODY-WEIGHT BIAS: INFLUENCE OF GENDER OF PARTICIPANTS AND TARGETS
}

\author{
Jason Nolan, Carol Murphy, and Dermot Barnes-Holmes \\ National University of Ireland, Maynooth
}

\begin{abstract}
The Implicit Relational Assessment Procedure (IRAP) and explicit measures were used with 21 college students to determine if body weight of target stimuli (photographic images of slim and overweight individuals) influenced perceptions of intelligence. The explicit measure was a computerized adaptation of the Anti-Fat Attitudes Questionnaire designed to reduce participant error. A Covert Perception Task was developed as an additional explicit measure in which participants rated the likelihood of success of slim and overweight job applicants. Correlational analyses were used to determine if disparity between current and idealized body mass index (BMI) influenced responding. Statistical analyses were applied to data to determine influence exerted by the gender of participants and/or by the gender of the portrayed target stimuli. Findings are discussed in relation to pro-slim versus anti-fat bias and gender differences in body-weight bias related to participants and targets.
\end{abstract}

Key words: IRAP, Relational Frame Theory, implicit responding, intelligence, body-weight bias

The World Health Organization has described obesity as a worldwide epidemic (WHO, 2000). It is estimated that approximately 315 million people worldwide fall into the category of obesity (Caterson \& Gill, 2002). Prevalence rates of obesity are higher in developed countries, with the Western world accounting for a significantly high proportion of obese individuals (James, Leach, Kalamara, \& Shayeghi, 2001). Obesity is associated with numerous health difficulties, such as diabetes, hypertension, asthma, and arthritis (Mokdad et al., 2001). In addition to physical health problems, there are also a number of psychological problems related to obesity, such as depression, bodyimage disturbances, and low self-esteem (Freidman et al., 2005). Physical and psychological difficulties in obesity are likely to be exacerbated by social stigma associated with being overweight.

Negative attitudes toward overweight individuals have been measured in many different ways. The traditional approach to measuring negative body-weight bias is the administration of a questionnaire, where the participant is generally asked a number of questions related to their attitudes toward overweight people (see Crandall, 1994).

Correspondence concerning this article should be addressed to Carol Murphy, Department of Psychology, John Hume Building, National University of Ireland, Maynooth, Maynooth, Co Kildare, Ireland; E-mail: Carol.A.Murphy@nuim.ie 
A second approach is to set up a covert behavioral experiment, designed to assess bodyweight bias by measuring the behavioral responses of participants (O'Brien, Latner, Halberstat, Hunter, Anderson, \& Caputi, 2008). For example, in the study by O’Brien et al., participants were given a mock curriculum vitae (CV) that included a photograph of either a slim or an overweight individual. The experimental group received the mock CV with a picture of an overweight individual, whereas the control group was given the same CV with a picture of a slim individual. In one behavioral measure, participants were told that the individual in the picture would sit in a particular chair in the same room with the participant. The researchers then monitored where each participant sat relative to the chair that was indicated for the individual in the picture. Participants who received the picture of the overweight individual sat farther from the chair than those in the control group. Thus, a behavioral bias against overweight individuals was observed.

The questionnaire is a useful and convenient method of obtaining information for analysis from a large sample of participants, compared to more time-consuming experimental behavioral studies, such as that conducted by O'Brien et al. (2008). However, surveys may not be the most appropriate method of analysis when the subject matter is controversial in nature, for example, when measuring attitudes relating to social prejudice (Dovidio \& Fazio, 1992). One of the major shortfalls of self-report measures such as questionnaires when probing socially sensitive issues is that it is possible that participants may not respond honestly, as they may not wish to admit to possessing negative attitudes toward socially vulnerable groups. A research approach designed to overcome the difficulties related to a social desirability bias is the Implicit Association Test (IAT; Greenwald, McGhee, \& Schwartz, 1998). The IAT is a computerized test procedure that measures what is termed implicit attitudes, because any bias exposed is implicit in the nature of participant responding, rather than in explicit statements and participants' reported agreement or disagreement. Specifically, whereas questionnaires and other self-report methods may be referred to as "explicit measures," implicit bias is not reported by the participant but is interpreted by the researcher via response latency data. The basic premise of implicit attitude testing is that participants will respond faster to statements that are consistent with their beliefs than to statements that are inconsistent with their beliefs. For example, if the IAT program presents paired associations such as flower-pleasant and flower-unpleasant, predictions are that participant responding will be quicker to agree with the flower-pleasant association than with the flower-unpleasant association. Response latencies are measured by the IAT program and, indeed, Greenwald et al. (1998) found that participant response latencies were shorter for the flower-pleasant association. The difference between average response latencies for consistent trials (e.g., flower-pleasant) compared with average response latencies for inconsistent trials (e.g., flower-unpleasant) is termed the IAT effect, and is interpreted as an implicit bias. The IAT has been used to examine many socially sensitive issues, such as racial and age stereotypes, and studies that have compared explicit and implicit measures on sensitive issues frequently report that participants show a greater degree of bias in implicit tests compared to explicit tests (Teachman, Gapinski, Brownell, Rawlins, \& Yeyaram, 2003).

The IAT has also been used to examine attitudes toward obesity (Ahern \& Hetherington, 2006; Gapinski, Schwartz, \& Brownell, 2006; Greenwald et al., 1998; Schwartz, Vartanian, Nosek, \& Brownell, 2006). In a typical IAT measuring obesity bias, participants are required to match pictures of slim and overweight people with either positive or negative words. It is generally found that participants respond faster when pairing slim people with positive words and overweight people with negative words than they do when pairing slim people with negative words and overweight people with positive words. As in other IAT studies, the difference in reaction time (response latency) is assumed to provide an indication of implicit bias.

Although the IAT has been very useful in that it is sensitive to biases that may not be detected via questionnaire enquiry, a limitation of the IAT is that it does not provide 
detail regarding the direction of bias. For example, in the case of attitudes toward overweight individuals, a strong IAT effect may indicate a favorable bias toward slim individuals, or an unfavorable bias toward overweight individuals, but this remains unknown because the IAT does not indicate the relative strength of pro-slim or anti-fat bias (De Houwer, 2002). The recent development of another implicit test has addressed this issue, however, and this research methodology is known as the Implicit Relational Assessment Procedure (IRAP; Barnes-Holmes et al., 2006). For example, a recent study of implicit pro-slim/anti-fat attitudes compared findings using the IRAP, the IAT, and explicit measures and found that both implicit measures detected higher levels of bias than the explicit measure; however, the IRAP data provided additional information in that analysis of the trial type data indicated a greater pro-slim than anti-fat bias (Roddy, Stewart, \& Barnes-Holmes, 2010).

The IRAP is a computerized program that is freely available to researchers (Software and sample instructions are available from http://irapresearch.org/downloads-andtraining/). The IRAP is similar to the IAT, in that it measures response latencies; however, unlike the IAT, it provides more information about the direction of bias, as described in the study by Roddy et al. (2010). In a typical IRAP measuring obesity, participants are presented with a positive or negative word along with a picture of a slim or overweight individual. In this way, four different trial types can be presented: positive-slim, positivefat, negative-slim, and negative-fat combinations. Two response options are also presented, such as "similar" and "opposite" or "true” and "false.” Detailed instructions are provided to participants prior to commencing the IRAP program. In the first block of trials, participants are required to respond in a pro-slim/anti-fat manner, for example, responding "similar" to positive-slim and negative-fat trial types and "opposite" to positive-fat and negative-slim trial types. These may be termed consistent trials, as the relations are possibly consistent with verbal relations established in the wider community. In the next block of trials, participants are required to respond in a pro-fat/anti-slim manner, this time responding "similar" to the positive-fat and negative-slim trial types and "opposite" to the positive-slim and negative-fat trial types. These are termed inconsistent trials. Response latencies are recorded for each of the four trial types, under each condition (consistent or inconsistent), thus providing four potential measures of bias. This allows for the researcher to identify both the extent and the direction of the implicit bias by subtracting the calculated average latency scores for consistent trials from those of inconsistent trials to determine if responding was faster in consistent trials, indicating bias in favor of the proposed consistent relations.

The IRAP has been shown to be an effective measure of determining implicit bias. A number of studies have tested the validity of the IRAP by comparing it to the IAT (BarnesHolmes, Murtagh, Barnes-Holmes, \& Stewart, 2010; Barnes-Holmes, Waldron, BarnesHolmes, \& Stewart, 2009). The reliability of the IRAP has also been tested, and it has been shown to be resistant to faking (McKenna, Barnes-Holmes, Barnes-Holmes, \& Stewart, 2007). For this reason, the IRAP is considered useful for measuring attitudes toward a sensitive or controversial topic (Barnes-Holmes, Murphy, Barnes-Holmes, \& Stewart, 2010). The IRAP has also been shown to offer a greater contribution to predictive validity toward behavioral intentions than the IAT (Roddy et al., 2010).

The IRAP pro-slim/anti-fat study by Roddy et al. (2010) used photographs of slim and overweight men and women, previously used by Brochu and Morrison (2007). These photographs were matched for levels of attractiveness. The target words used in the study consisted of six positive words (desirable, active, disciplined, attractive, healthy, and good) and six negative words (undesirable, lazy, sloppy, ugly, ill, and bad). However, including the attributes healthy and active may be somewhat problematic for a study of this type. Specifically, it may be reasonable to assume that a slim individual is more healthy and active because, for example, the medical community tells us frequently that being overweight is unhealthy and that inactivity contributes to being overweight. Therefore, speedier associations of these words with slim rather than overweight people may not be 
indicative of negative bias toward the overweight (Schwartz et al., 2006). What we consider a negative bias might be described as an unflattering assessment or judgment that is unjustified on the basis of the evidence available. Thus, to avoid potential confounding influence resulting from use of attributes such as healthy or active in the current study, these terms have been avoided, and positive and negative attributes that are directly related to intelligence have been carefully selected.

The current study is the first of its type to seek to measure implicit attitudes towards the intelligence of the overweight, to examine pro-slim and anti-fat bias with a sample of psychology students. For the photographic stimuli in the current study, "before” and "after" images were used of the same individual, having undergone significant weight loss. It was expected that this technique would offer increased control and avoid confounding effects that may arise from using pictures of different people (e.g., related to different levels of attractiveness), as only the person's weight changes. It was predicted that an IRAP effect would be demonstrated in the context of an intelligence evaluation and that in accordance with previous studies, the effect would be more pro-slim than anti-fat.

The current study also employed a new Covert Perception Task developed by the first author. The Covert Perception Task was so called because participants were not overtly told prior to the task that it was related to body weight. This method is similar to the Person Perception Task (PPT), developed by Brochu and Morrison (2007). Participants were required to read a short description of a fictional individual. Four versions of the description were created, with each differing only in terms of the gender and weight of the individual. Participants were then given a short questionnaire relating to the perceived intelligence and job prospects of the person in the description. The concept of intelligence was not explicitly linked with the weight of the individual, but researchers wished to test whether the described weight of the individual would influence participant ratings. Would participants who received the "slim" version of the description rate the individual more favorably in terms of job prospects and intelligence than participants who received the “overweight” version? Yes.

Crandall's (1994) Anti-Fat Attitudes Questionnaire was used as the explicit measure in the current study. This scale incorporates 13 items in total and consists of three subscales: Dislike (7 items), Fear of Fat (3 items), and Willpower (3 items). An electronic version of the scale was developed by the first author in the current study to reduce the possibility of participant error. It was expected that both the Covert Perception Task and the implicit measure (IRAP) would demonstrate higher body-weight bias than the explicit self-report (questionnaire) measure. Gender differences in body-weight bias have not been extensively studied, and some studies that have employed implicit measures have only used female participants (Ahern \& Hetherington, 2006; Gapinski et al., 2006). However, important information may be lost in this approach because previous research has identified that males may show greater body-weight bias than females (Brochu \& Morrison, 2007; Crandall, 1994; Lewis, Cash, Jacobi, \& Bubb-Lewis, 1997; O’Brien, Hunter, Halberstadt, \& Anderson, 2007). These findings that males show greater anti-fat bias are interesting and perhaps counterintuitive, in that males are generally thought to be less affected by negative body-weight bias (O’Brien et al., 2007). Another aspect that remains currently unclear is whether participants respond differently, in terms of body-weight bias, to male and female targets (Hebl \& Turchin, 2005; Puhl \& Brownell, 2006). Thus, an additional aim of the current research study was to examine this question in the context of male and female perceptions of intelligence in slim and overweight males and females. Research questions were as follows: (1) Will participants demonstrate implicit body-weight bias in the context of intelligence evaluation, and will this bias will be primarily pro-intelligent-slim or anti-intelligent-fat? (2) Will participants demonstrate body-weight bias by rating overweight targets as less intelligent and less likely to gain employment, compared to normal weight targets? (3) Will gender differences be observed in terms of implicit bias, and will the gender of the participants 
and/or the gender of the target stimuli (photographs of male and female targets) exert influence? and (4) Will body-weight bias be observed on the Covert Perception Task and the implicit measure, but not on the explicit measure?

\section{Method}

\section{Participants}

A total of 21 participants completed the experiment. The sample consisted of 14 males and 7 females, with ages ranging from 17 to 55 years. Participants were recruited through a mixture of university students at the National University of Ireland, Maynooth, and a convenience sample (four participants) drawn from friends and family of the researcher. Participants were randomly assigned to four conditions in the first part of the experiment, and a repeated-measures design was used thereafter. In the implicit measure, three participants failed to pass the practice stage of the IRAP, resulting in their data being excluded from the experiment.

\section{Materials and Apparatus}

All four parts of the experiment were administered on a standard laptop (Sony Vaio, dual-core processor, running Windows 7).

Covert Perception Task (CPT). The CPT consisted of a written stimulus, which was a brief written description of a fictional individual, followed by a related five-item questionnaire (see Appendix A). Four different versions of the written description stimulus were used, with each varying in terms of the gender and weight of the individual in the description. The four stimuli versions were male normal weight, male overweight, female normal weight, and female overweight. Participants were randomly assigned to one of four conditions, with each condition receiving one of the four stimuli. The five-item scale was common to all conditions and contained general statements regarding the individual in the description. Participants were required to respond to the statement by means of a 5-point rating scale ( 1 = strongly disagree, 5 = strongly agree $)$.

Participant Information Questionnaire (PIQ). The Participant Information Questionnaire is a software program written by the first author specifically for the current experiment. This program obtained relevant information about the participants, such as gender, age, and height. The PIQ also contained two measures of body mass index (BMI), one for the participant's current BMI and one for their ideal BMI. The method used to obtain BMI information was to adopt a selection of computer-generated human figures with corresponding BMI values ranging from 18.5 to 40. Participants selected two images, one that was the closest match to their current BMI and one that was their ideal BMI body type (see Appendix B).

Explicit Anti-Fat Attitudes Questionnaire (AFA). The AFA was developed by Crandall (1994) in order to assess explicit attitudes toward overweight people. This scale incorporates 13 items in total and consists of three subscales: Dislike (7 items), Fear of Fat (3 items), and Willpower (3 items). An electronic version of the AFA was developed by the first author in the current study to reduce the possibility of participant error. All of the items on the scale consisted of statements such as "I do not like fat people," and the participants were required to indicate the extent to which they either agreed or disagreed with the statement using a 10 -point scale $(0=$ true, $9=$ false $)$.

Implicit Relational Assessment Procedure (IRAP). The IRAP (Barnes-Holmes et al., 2006) is a computer application designed to measure implicit attitudes. The IRAP presents the participants with a label stimulus (e.g., intelligent), a visual target stimulus (e.g., a photograph of a slim person), and response options (e.g., "similar" or "opposite”). The label stimuli consisted of six positive words (intelligent, successful, clever, brainy, smart, and bright) and six negative words (stupid, dumb, foolish, brainless, dim, and dull). Digital photographs of thin and overweight people were used as the target stimuli. The 
images consisted of three men and three women and showed each of the individuals before and after they had lost a significant amount of weight. This gave a total of 12 target stimuli. Each image pair was carefully selected to match on factors such as facial expression, clothing, and pose in order to control as many variables as possible. The investigator replaced the background of each image with a white fill in order to reduce any distracting features in the photographs. All images were sourced from a Google image search and are available from the author on request. The response options used were "similar" and "opposite," which the participant selected using either the "d" or "k" keys on the laptop keyboard.

\section{Experimental Design}

The experiment consisted of four phases. In the first phase (CPT), a between-subjects design was used. Participants were randomly assigned to one of four experimental groups in which there were two independent variables (IVs): gender of the target stimulus and weight of the target stimulus. Each IV has two levels: male/female and normal weight/ overweight, respectively. The dependent variable (DV) was the total score of the five-item questionnaire. The second phase of the study used a correlational design. The third phase used a questionnaire. A between- and within-subjects design was used in the fourth phase (the IRAP). Gender was the between-groups variable. Trial type was the within-subject variable. The trial type IV consisted of two levels: intelligent-slim/unintelligent-fat (consistent trials) and unintelligent-slim/intelligent-fat (inconsistent trials). All participants were exposed to both levels of the IV. The DV measured was the average time taken by the participant to select the correct response option, in other words, the response latencies.

\section{Ethical Issues}

Due to the sensitivity of the subject matter (i.e., body weight) in the experiment, special care was taken to ensure that an ethical approach was in place prior to the experiment. Briefing was provided at two separate occasions for all participants. Prior to the CPT, participants were informed that the experiment was designed to measure implicit attitudes, and at this point, they were not informed of the focus on perceptions about normal or overweight people. This was to ensure that the CPT was not confounded, and this mild deception was thought to be justified because (a) all data collected were treated confidentially and (b) subsequent to the CPT procedure, participants were fully debriefed and fully informed about the focus of the study. Participants were then told by the investigator that if they wished, they could consider their decision to participate over a 24-hour "cooling off" period. The participants were advised that all data would be treated confidentially and that the investigator would be available to answer questions during or after the study. A signed consent form was obtained for all participants in the experiment, and this explained that participants could withdraw their participation at any point. Following the experiment, a full debriefing was carried out.

\section{Procedure}

The study consisted of four phases, which were all completed on a standard laptop computer with external mouse for ease of use. All participants completed the four stages sequentially and were seated at a desk in a quiet room. The investigator provided verbal instructions before each phase, and the participants were also advised to follow the directions on-screen. The researcher left the room during each task to minimize distraction.

Covert Perception Task (CPT). In Phase 1, participants completed the CPT. At the beginning of this task, participants were presented with the following instructions: 
Thank you for participating in the experiment. This experiment consists of four parts and takes approximately 20-30 minutes to complete. In the first three parts, you will be asked a series of questions. Please answer these as honestly as possible. The fourth part of the experiment involves a reaction time task. Please ask the researcher if you have any queries during the experiment.

At the next screen, participants were presented with one of four character descriptions, depending on which group they were assigned to. The participants in the "male overweight” condition received this description:

Gerry is a 22-year-old man. He is $5 \mathrm{ft} 8$ in $(173 \mathrm{~cm})$ tall, weighs $250 \mathrm{lb}$ (113 $\mathrm{kg}$ ), has dark hair, and brown eyes. Gerry works part-time in a busy fastfood restaurant. He is currently in his first year of an arts degree in NUI Maynooth. He enjoys socialising with friends and goes for nights out on most weekends. Gerry’s friends describe him as generally happy and easy going. In time Gerry would like to teach in a secondary school.

Only the gender and weight of the individual were changed between the groups. For example, Gerry was replaced with Geraldine, he with she, and so on. The weight of the individual was either 150 or $250 \mathrm{lb}$, depending on the condition, to depict either an obese person or a person of normal weight. Appendix A provides all four descriptions. After participants read the description, the following instructions were presented:

Next, please answer the following questions using the slider to indicate your answer. Remember please answer honestly using your "gut” feeling.

The participants were then given a five-item questionnaire relating to the individual in the description. Each question consisted of a general statement, such as "The person in the description is intelligent.” Participants were required to respond using a six-point scale ( 1 = agree strongly, 6 = disagree strongly). The CPT then advised the participant to call the researcher upon completion.

Participant Information Questionnaire (PIQ). Following a brief verbal instruction by the researcher, the participants were advised to proceed with the second phase of the experiment. In this phase, participants used the on-screen program to provide details of their gender, age, height (height could be provided in centimeters or feet and inches), current weight, and ideal weight. BMI measures were obtained using a computer-generated pictorial measure, and the program required participants to select two BMI images - one that resembled their current BMI and one that resembled their perceived ideal BMI. Data were subsequently analyzed for correlations with other measures to determine if disparity between current and ideal BMI influenced responding on other measures.

Anti-Fat Attitudes Questionnaire. Following completion of the PIQ, participants completed the third phase of the experiment. This involved the completion of the Anti-Fat Attitudes Questionnaire (Crandall, 1994). This scale was incorporated into the software application. Participants followed the on-screen instructions and were told to call the researcher once Phase 3 was completed.

IRAP. In Phase 4 of the experiment, participants were required to complete the IRAP (Barnes-Holmes et al., 2006). Before commencement of the IRAP, participants received both verbal and written instructions. The structure of the IRAP was explained, and onscreen examples were provided for the different trial types. The participants were advised to follow the on-screen instructions during the course of the task. At this stage, participants were also made aware that the conditions for gaining a correct response would change throughout the test, as per the on-screen instructions (e.g., for consistent trials and inconsistent trials). The IRAP practice blocks were started once the researcher was satisfied that the participants fully understood the task. 
The IRAP presented the participants with a series of trials grouped into blocks. Each trial consisted of one sample word (e.g., intelligent, dim) at the top of the screen. Directly below this was one of the photographic stimuli with either a slim individual or an overweight individual. At the bottom left and right of the screen were two response options, "similar" and "opposite" (note that the relative position of the response options was counterbalanced throughout the test). Directly above each response option was the text "Press ' $d$ ' for" (over the left response option) and "Press ' $k$ ' for" (over the right response option). Thus, the participants pressed the " $\mathrm{d}$ " key to select the left response option and the "k" key to select the right response option. Each block consisted of 24 trials in total. The stimuli presented in each trial were selected in a quasi-random fashion. Each photograph was presented twice, for example, once with an "intelligent" sample word and once with an "unintelligent" sample word. This created four trial types: intelligent-slim, intelligent-fat, unintelligent-slim, and unintelligent-fat.

The IRAP began with a number of practice test blocks to allow the participant to become familiar with the procedure. Half of the participants were informed that during the first practice block, the correct responses were consistent with an intelligent-slim/ unintelligent-fat bias. In other words, the participants in this condition should select the response option "similar" for intelligent-slim and unintelligent-fat trial types and "opposite” for intelligent-fat and unintelligent-slim trial types. The remaining participants were informed that the first practice block would require responses that were inconsistent with an intelligent-slim/unintelligent-fat bias. In this condition, the correct response for intelligent-slim and unintelligent-fat trial types was "opposite," and the correct response for intelligent-fat and unintelligent-slim trial types was "similar."

Before the first practice block, the following on-screen instructions were presented to the participant:

If you make an error, you will see a red ' $X$ ' below the stimulus-When this happens, you have to make the correct response to proceed. This is practice-Errors are expected. Press space bar to start.

Once the participant pressed the space bar, the first practice block commenced, and the first trial was presented. If the participant selected the correct response option, the screen would go blank for $400 \mathrm{~ms}$, and the next trial would be presented. Should the participant select the incorrect response option, a red X would appear below the target photograph. Once the participant selected the correct response option, the screen would go blank for $400 \mathrm{~ms}$ and then present the next trial. If the participant failed to select either response option after a 2,000-ms interval, the text "Too Slow" would appear under the target photograph. The function of this feedback was to prompt the participant to respond as quickly as possible. Once all 24 trials were completed, a feedback screen was presented that indicated the percentage of correct responses and the median response time in milliseconds for the block. The participant then pressed the space bar to proceed.

At the beginning of the second practice block, the following instructions were presented on-screen:

Important: During the next phase, the previously correct and wrong answers are reversed. This is part of the experiment. Please try to make as few errors as possible-In other words, avoid the red X.

This screen informed the participants that during the next block, they would have to reverse their response criteria in order to gain a correct response. In other words, participants in the consistent condition would now need to respond in an inconsistent manner, and participants who previously responded in an inconsistent manner switched to the consistent condition. The pre-practice block instructions were again presented onscreen, informing the participants to press the space bar to start the second practice block.

The IRAP required that participants reach a performance standard, where the 
percentage of correct score was higher than 80 and the median response time was lower than 2,000 ms. Once this criterion was achieved on both practice blocks, the participants began the test blocks. If the participant failed to reach the performance criteria, a feedback screen was presented. This informed the participants of their performance on the previous two practice blocks and reminded the participants about the required level of performance. The participant was then given two further attempts to pass the practice stage. If, after six practice blocks, the participant failed to reach the performance criteria, the test was stopped and the participant's scores were deleted.

Once the participant met the performance criteria, the IRAP proceeded to the test blocks. The following instructions were presented prior the start of the test blocks:

If you make an error, you will see a red ' $X$ ' below the stimulus-When this happens, you have to make the correct response to proceed. This is a testGo fast, making a few errors is ok. Press space bar to start.

The participant then completed six test blocks, alternating between the consistent and inconsistent conditions on each new block of trials. Once all test blocks were completed, a screen was presented to inform the participant that the experiment was finished and instructing him or her to call the investigator. Participants were then debriefed.

\section{Results}

\section{Covert Perception Task}

A one-way between-groups analysis of variance was conducted to compare scores on the CPT between the four experimental conditions (male overweight, male normal, female overweight, and female normal). For the overall group of male and female participants, there was no statistically significant difference between the conditions. There was a statistically significant difference on test scores between the overweight groups $(M=17$, $S D=1.93)$ and the normal groups $(M=12.5, S D=4.51)$ for male participants $(t[12]=2.56$, $p<.05, r=.59$ ), and no other significant differences were found.

\section{CPT-AFA-PIQ Correlations}

Pearson's two-tailed correlation tests were applied to the data. Average scores on the CPT for both overweight gender groups (male overweight and female overweight) were compared with scores on the AFA and the PIQ. A strong statistically significant correlation was found between total CPT scores and two of the AFA subscales: Fear of Fat $(r=-.76$, $n=11, p=.006)$ and Willpower $(r=.79, n=11, p=.004)$. The height of the participants was found to correlate with CPT scores $(r=.70, n=11, p=.016)$, total average AFA scores $(r=.56, n=21, p=.020)$; Dislike $(r=.52, n=21, p=.016)$, and Willpower $(r=.72, n=21$, $p<.0001)$. There was also a statistically significant correlation between the age of the participant and average scores on the AFA $(r=-.50, n=21, p=.020)$.

\section{Participant Information Questionnaire}

The overall average scores related to perceived current BMI reported by participants via the computer-generated images and related scale was calculated as 19.5. The overall average scores related to perceived ideal BMI calculated from the scale figures was 18.1. The average height reported by participants was $173 \mathrm{~cm}(S D=7.3)$. Males reported a higher average perceived current BMI than did female participants (males: $M=20.5$; females: $M=18.2$ ). The average ideal BMI reported by male participants was 18.36 , whereas the average ideal BMI reported by females was 17.6. Correlation tests were conducted with PIQ data, AFA data, and CPT data to determine any influence of disparity between perceived and ideal self-perception of BMI, but no correlation relevant to the PIQ test was detected. 


\section{Explicit Measure: The AFA}

The overall average score for the Anti-Fat Attitudes Questionnaire was 4.14 $(S D=1.16)$, with a range of scores of 0 to 9 . The scores for the subscales were 3.16 $(S D=1.79)$ for Dislike, $3.92(S D=2.64)$ for Fear of Fat, and $6.65(S D=1.64)$ for Willpower. Overall, this indicated a low explicit anti-fat bias. Male participants scored higher $(M=3.76, S D=1.78)$ than female participants $(M=1.96, S D=1.11)$ on the Dislike subscale $(t[19]=2.42, p<.05)$. Males also scored higher $(M=7.31, S D=1.02)$ than females $(M=5.33, S D=1.90)$ on the Willpower subscale $(t[19]=2.57, p<.05)$. Thus, scores on the AFA indicated a low anti-fat bias for male participants.

\section{IRAP}

The key measure of the IRAP was response latency. This was defined as the time taken by the participant to select the correct response option, following presentation of the trial. In order to account for individual differences in response time, the raw response latencies were transformed into D-IRAP scores (see detailed description in Barnes-Holmes et al., 2009). The D scores represent the difference between the mean response latencies for consistent and inconsistent trial blocks after the smaller mean latencies (usually consistent trial blocks) have been subtracted from the larger mean latencies (usually inconsistent trial blocks). This current procedure is an adaptation of the D-algorithm used by Greenwald, Nosek, and Banaji (2003). In this experiment, only the response latencies from the three test block pairs were used. The data were screened, and any latencies above 10,000 ms were removed from the data pool (this was to ensure that average response latencies were not unduly skewed). Data for each test block consisted of response latencies for the four trial types; this gave a total of 24 sets of response latencies. The 24 mean latencies were calculated from each trial type in each test block, and 12 standard deviations were also calculated for each trial type across each block pair. Difference scores were calculated for each trial type by subtracting mean latencies for consistent trials (intelligent-slim/unintelligent-fat) from mean latencies for inconsistent trials (intelligent-fat/unintelligent-slim) for each test block pair. Each difference score was divided by the corresponding standard deviation for the trial pair, giving 12 D-IRAP scores (four scores for each test block pair). These scores were then averaged over the three test block pairs, resulting in four D-IRAP scores representing each trial type (intelligent-slim, intelligent-fat, unintelligent-slim, and unintelligent-fat). Positive D-IRAP scores indicate an intelligent-slim/ unintelligent-fat bias, whereas negative D-IRAP scores indicated an intelligent-fat/ unintelligent-slim bias in implicit responding. D scores were subjected to statistical analyses.

\section{Overall D-IRAP Score}

Figure 1 depicts the D-IRAP scores for each trial type for 21 male and female participants. The overall mean D-IRAP score was statistically significant and different from $0(M=.12, S D=.22, t[20]=2.4, p<.05, r=.48)$. One-sample planned $t$ tests were conducted to determine if the D-IRAP scores for each of the trial types for both groups were significantly different from 0 . Analysis of individual trial types indicated that the intelligent-slim trial type, and the unintelligent-slim trial type were statistically significant different from $0(M=.27, S D=.52, t[20]=2.4, p<.05, r=.48 ; M=.17$, $S D=.25, t[20]=3.0, p<.05, r=.56)$. The scores for the intelligent-fat and unintelligentfat trial types were not statistically different from 0 . These findings suggest that participants readily agreed that slim individuals are intelligent and did not readily agree that slim people are unintelligent. 


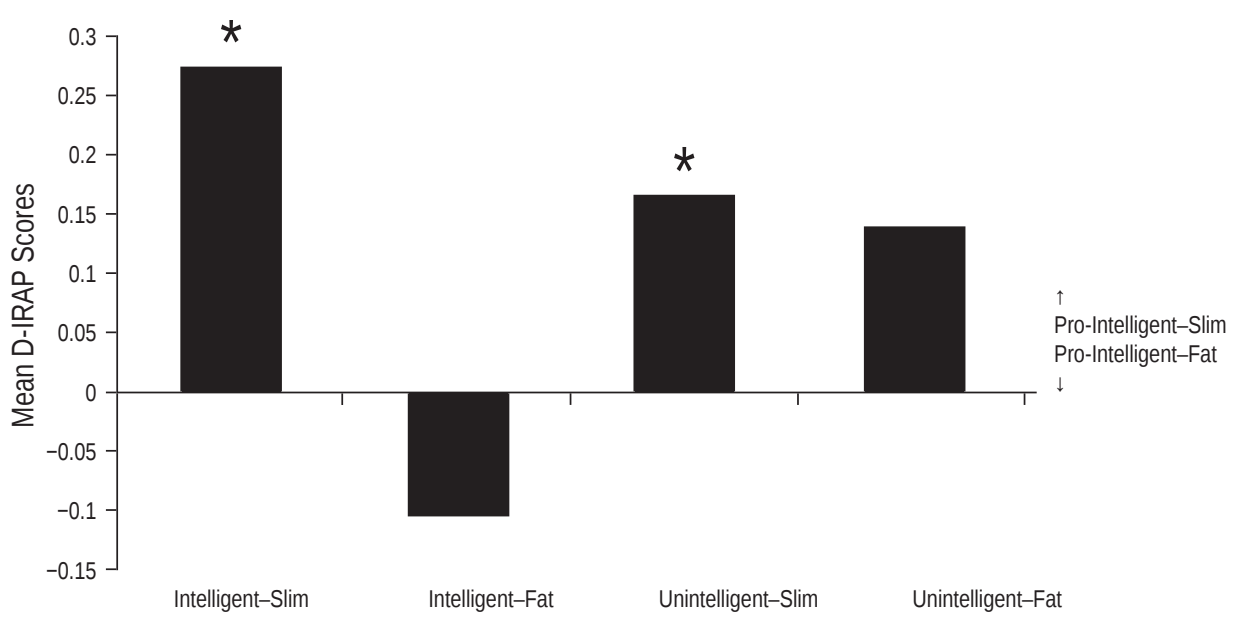

IRAP Trial Type

Figure 1. Overall mean D-IRAP scores. Bars in the positive direction indicate a prointelligent-slim bias, whereas bars in the negative direction indicate a pro-intelligent-fat bias. Statistical significance was observed for the intelligent-slim and the unintelligent-slim trial types. ${ }^{*} p<.05$

\section{D-IRAP Scores and Gender of Participants}

Figure 2 presents the D-IRAP scores split by the gender of participants ( $n=14$ males, $n=7$ females). Overall D-IRAP scores were statistically significant for male participants $(M=.18, S D=.25, t[13]=2.7, p<.05, r=.60)$ but not for female participants. D-IRAP scores were significantly different from 0 for the intelligent-slim, unintelligent-slim, and unintelligent-fat trial types for male participants $(M=.42, S D=.52, t[13]=3.02, p<.05$, $r=.64 ; M=.21, S D=.26, t[13]=3, p<.05, r=.64 ; M=.23, S D=.36, t[13]=2.4, p<.05$, $r=.55)$. Male participants showed a relatively strong pro-slim IRAP effect for the intelligent-slim trial type and a moderate pro-slim IRAP effect on the unintelligent-slim and intelligent-fat trial types. No trial types were statistically significant from 0 for female participants.

\section{D-IRAP Score Analysis and Gender of Target Stimuli}

The current IRAP procedure used photographic images of overweight and slim males and females as target stimuli. D-IRAP scores were calculated for each target gender type separately. The D-IRAP scores for each target gender type are presented in Figure 3. The overall D-IRAP score when male target stimuli were used was statistically different from $0(M=.14, S D=.25, t[20]=2.46, p<.05, r=.48)$. In addition, trial type data showed that intelligent-slim, intelligent-fat, and unintelligentfat trial types were all statistically different from 0 for male target stimuli $(M=.37$, $S D=.54, t[20]=3.15, p<.05, r=.58 ; M=.29, S D=.56, t[20]=-2.38, p<.05$, $r=.47 ; M=.24, S D=.37, t[20]=3.0, p<.05, r=.56)$. Participants showed a strong pro-slim IRAP effect for the intelligent-slim trial type, a strong pro-fat IRAP effect for the intelligent-fat trial type, and a strong anti-fat IRAP effect for the unintelligentfat trial type. In other words, participants readily agreed that slim individuals are intelligent and that fat individuals are intelligent, and they failed to readily agree that fat individuals are unintelligent. Neither the overall IRAP effect nor individual trial type D-IRAP scores were statistically significant from 0 when female target photographic stimuli were presented. 


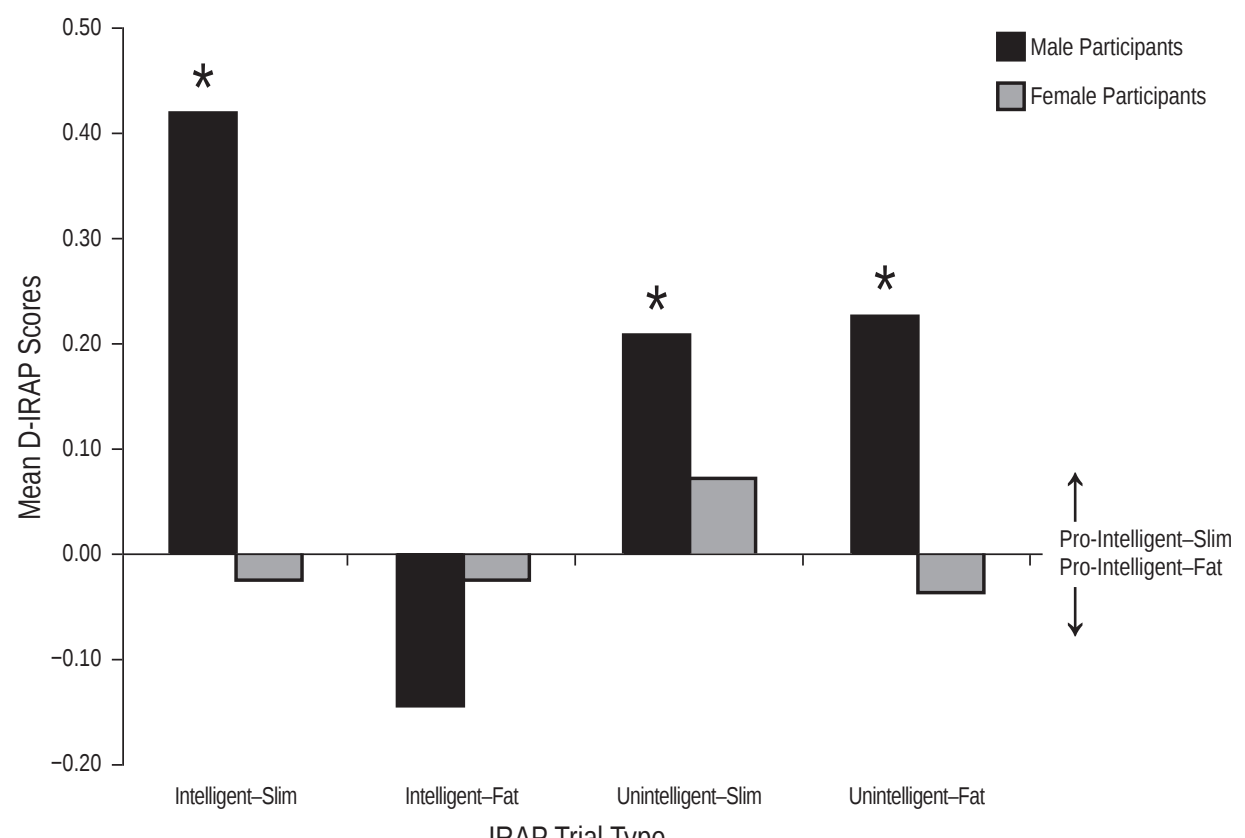

IRAP Trial Type

Figure 2. Mean D-IRAP scores for male and female participants. Bars in the positive direction indicate a pro-intelligent-slim bias, whereas bars in the negative direction indicate a pro-intelligent-fat bias. Statistical significance was observed for male participants on the intelligent-slim, unintelligent-slim, and unintelligent-fat trial types. ${ }^{*} p<.05$.

\section{D-IRAP Scores Analysis With Gender of Participant and Target Stimuli Combined}

D-IRAP scores were calculated for trials with male and female target stimuli and split by the gender of the participant. No statistically significant IRAP effects were observed with female participants. Figure 4 presents the D-IRAP scores for the male participants. In relation to male participants, total D-IRAP scores for both male and female targets were statistically different from $0(M=.18, S D=.26, t[13]=2.66, p<.05, r=.59 ; M=.18$, $S D=.29, t[13]=2.32, p<.05, r=.54)$. Analysis of the trial types for male participants revealed statistical significance for the male intelligent-slim, male intelligent-fat, male unintelligent-slim, and female intelligent-slim trial types $(M=.52, S D=.55, t[13]=3.52$, $p<.05, r=.70 ; M=.36, S D=.54, t[13]=2.44, p<.05, r=.56 ; M=.25, S D=.34$, $t[13]=2.77, p<.05, r=.61 ; M=.36, S D=.59, t[13]=2.40, p<.05, r=.55)$. Male participants indicated pro-slim IRAP effects on male intelligent-slim, female intelligentslim, and male unintelligent-slim trial types and a pro-fat IRAP effect for the male intelligent-fat trial type. In summary, male participants readily agreed that slim males and slim females are intelligent and that fat males, but not fat females, are intelligent. Male participants failed to readily agree that slim males are unintelligent.

\section{Implicit-Explicit Correlations}

Pearson's two-tailed correlation tests were conducted with implicit and explicit data. There were statistically significant correlations between average scores on the AFA and total IRAP effect $(r=.45, n=21, p=.039)$. AFA scores also correlated with the intelligent-slim trial type ( $r=.46, n=21, p=.035)$ and the unintelligent-fat trial type $(r=.44, n=21, p=.046)$. Scores on the Willpower subscale correlated with total IRAP 


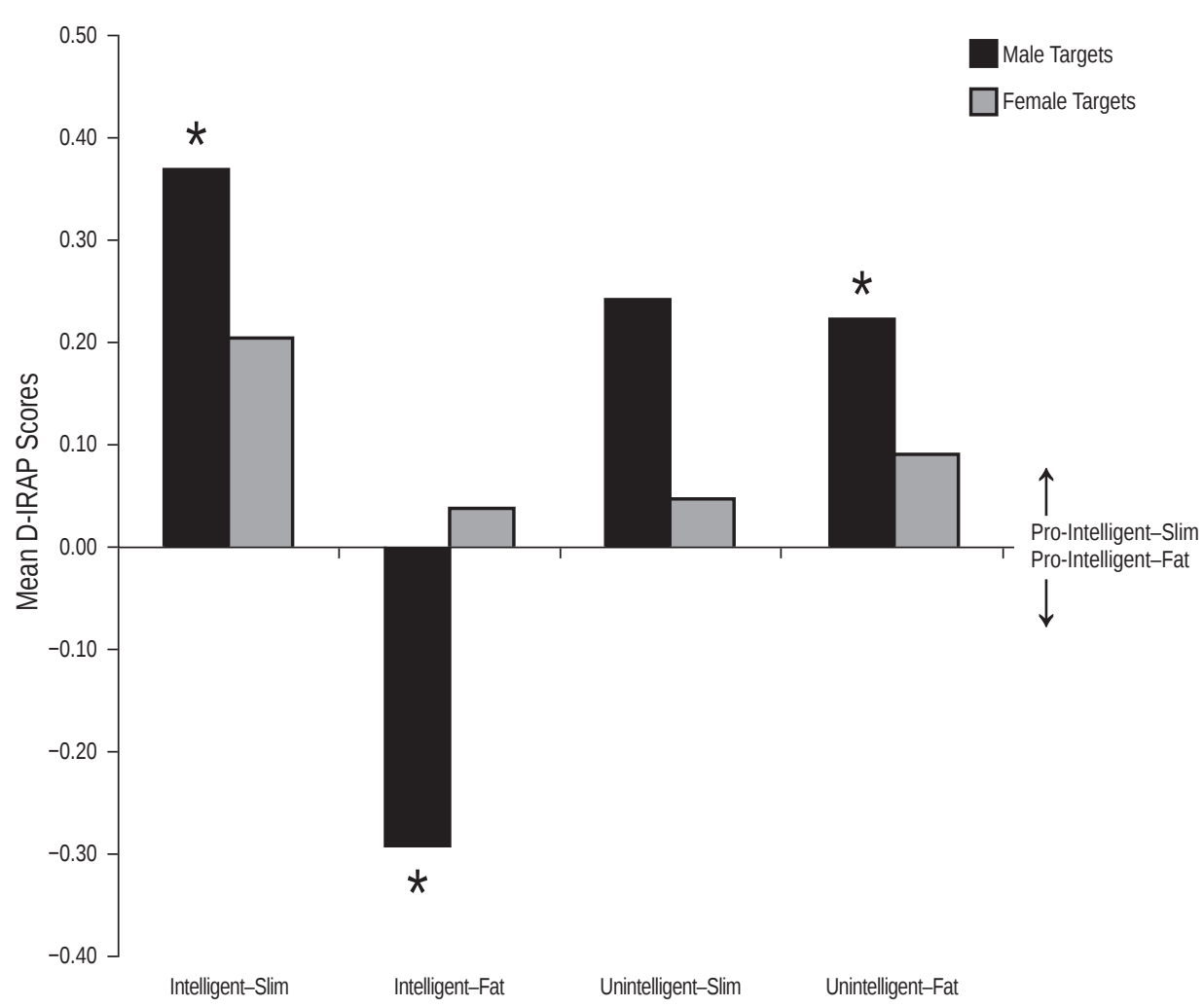

IRAP Trial Type

Figure 3. Mean D-IRAP scores for male and female photographic target types. Bars in the positive direction indicate a pro-intelligent-slim bias, whereas bars in the negative direction indicate a prointelligent-fat bias. Statistical significance was only observed on the photographic images of men. The intelligent-slim and the unintelligent-fat trial types showed a strong pro-intelligent-slim bias, whereas the intelligent-fat trial type indicated a strong pro-intelligent-fat bias. ${ }^{*} p<.05$.

effect $(r=.45, n=21, p=.039)$ and the unintelligent-fat trial type $(r=.51, n=21$, $p=.018)$. Implicit-explicit correlations were analyzed with male targets, resulting in a relationship between BMI difference (difference of current and ideal BMI) and the male unintelligent-fat trial type $(r=.49, n=21, p=.025)$. Female target analysis resulted in statistically significant correlations between average AFA scores and total IRAP effect $(r=.49, n=21, p=.025)$ and female intelligent-slim trial type $(r=.55, n=21, p=.01)$. Scores on the female unintelligent-fat trial type correlated with the Willpower subscale $(r=.53, n=21, p=.013)$.

\section{Discussion}

The primary question was whether participants would demonstrate implicit bodyweight bias in intelligence evaluation of slim and overweight individuals. Specifically, predictions were that participants ( $n=21$ college students) would perceive slim individuals as more intelligent than overweight individuals. The overall IRAP data supported this hypothesis, and this finding is consistent with previous research on implicit body-weight bias (Ahern \& Hetherington, 2006; Brochu \& Morrison, 2007; Gapinski et al., 2006; O’Brien et al., 2006; Schwartz et al., 2006; Teachman \& Brownell, 2001). Furthermore, it was postulated that the direction of the implicit bias would be favorable to intelligent-slim, rather than to unintelligent-fat, in accordance with previous research that found an overall 


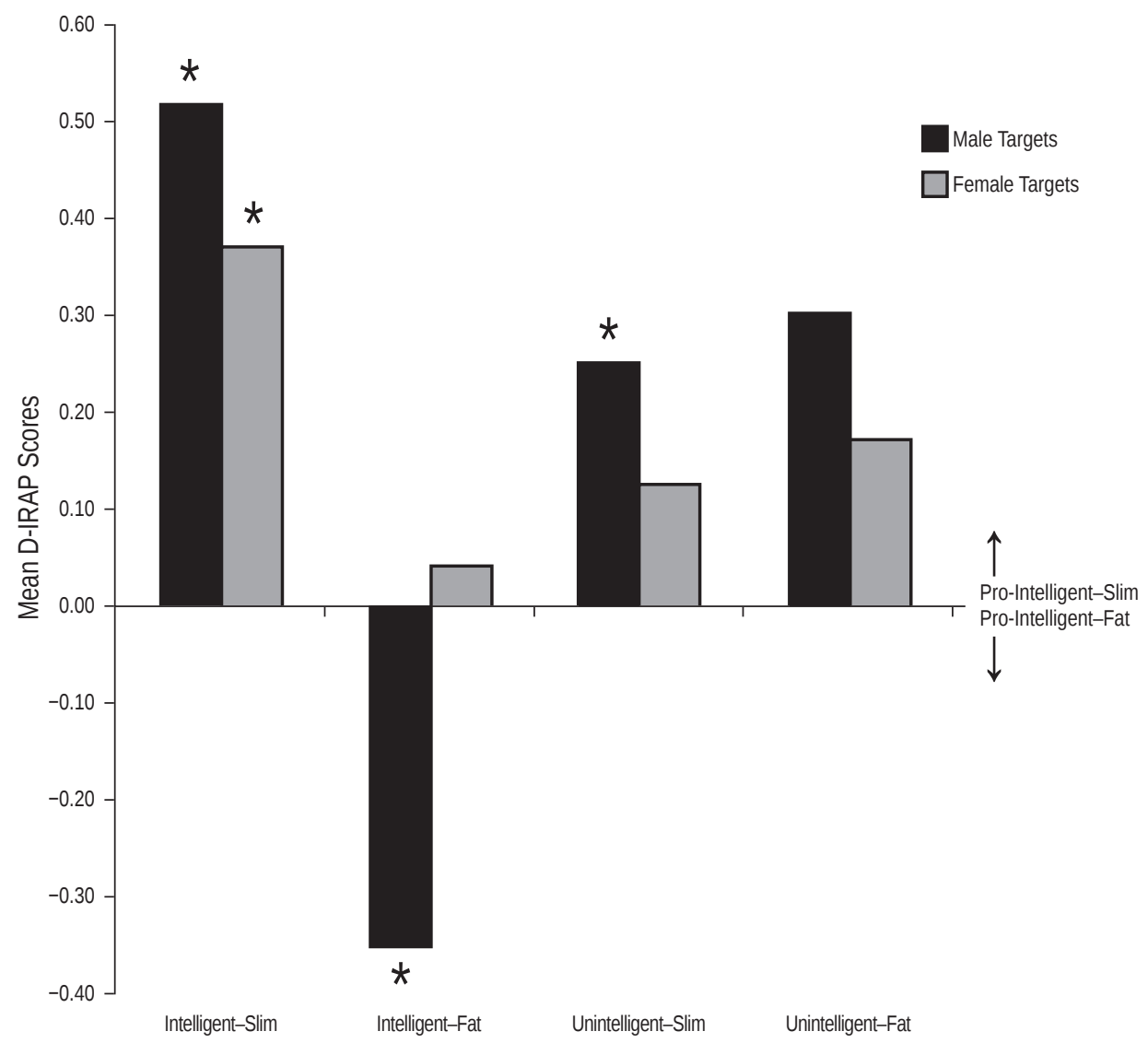

IRAP Trial Type

Figure 4. D-IRAP scores analyzing gender of target type, for male participants only. Bars in the positive direction indicate a pro-intelligent-slim bias, whereas bars in the negative direction indicate a pro-intelligent-fat bias. Male participants revealed strong implicit pro-intelligent-slim bias on the intelligent-slim trial type, for both male and female photographic targets. Moderate prointelligent-slim bias was also observed for male participants on the unintelligent-slim trial types. On the intelligent-fat trial type, males displayed a moderate pro-intelligent-fat bias toward male photographs. ${ }^{*} p<.05$.

pro-slim, rather than anti-fat, bias using a similar implicit measure (e.g., Roddy et al., 2010). The findings from this study were in accordance with this hypothesis.

Gender difference effects were also predicted in the implicit measure, based on previous findings of greater anti-fat bias for male participants compared to female participants (Brochu \& Morrison, 2007). Unlike previous research, the current study sought to test for gender differences in terms of both participant gender and the gender of the target stimuli (individuals portrayed in photographic images). Significant gender differences were observed when IRAP data were analyzed in terms of gender of participants. Specifically, most of the implicit bias was attributed to the male participant group, with no significant bias evident in data from the female group. The IRAP data also indicated that the gender of the photographic target stimuli was influential in the amount of implicit bias observed. Specifically, most of the implicit bias was focused on the male targets, and not the female targets. In brief, male participants showed greater favorable intelligence-slim bias toward male targets compared to female targets during IRAP, whereas IRAP results from female participants failed to show significant levels of bias. 
Thus, overall, the findings supported the hypotheses regarding gender effects in terms of implicit bias.

The current study extends previous implicit body-weight bias research because much of the previous research on body-weight bias has not investigated gender difference effects related to participants or target stimuli. Many studies that have used the IAT as the implicit measure of anti-fat attitudes have only used female participants (e.g., Ahern \& Hetherington, 2006; Gapinski et al., 2006), or have used a high proportion of female participants compared to male participants (Schwartz et al., 2006). Further research is undoubtedly required to clarify matters related to body-weight bias; for example, as noted by Roddy et al. (2010), the bias shown in previous IAT studies cannot be assumed to be anti-fat, and may instead represent a pro-slim bias. These matters will need to be teased out carefully, as will the matter of gender differences related to participants and target stimuli. The IRAP data indicating an implicit pro-slim rather than anti-fat bias are interesting, and the fact that the IRAP can detect the direction of the bias makes the procedure more informative than other implicit measures, for example, the IAT. From an RFT perspective, it might be expected that relating slim-intelligent-true would entail also relating overweight-intelligent-false (e.g., if "slim" and "overweight" participate in a relational frame of opposition). However, the issue appears to be more complicated, and thus it seems likely that multiple types of relational responding are involved when we consider implicit bias (see also Barnes-Holmes, Barnes-Holmes, Stewart, \& Boles, 2010, for a full discussion of automatic [implicit] responding versus relational elaboration and coherence). It might be speculated, for example, that conflicting relations in a relational network could exert influence. For example, an additional prejudice-bad relation might undermine the overweight-intelligent-false relation that may derive from slim-intelligent-true (e.g., additional relational responses may result in the formation of a coherent relational network; see Hughes, Barnes-Holmes, \& De Houwer, 2011). However, further research will be required to elucidate such matters.

The current IRAP data showing a greater negative bias toward the overweight for male participants compared to female participants are consistent with findings in previous research analyzing explicit data (Brochu \& Morrisson, 2007; Crandall, 1994; Lewis, Cash, Jacobi, \& Bubb-Lewis, 1997; O’Brien et al., 2007). However, although explicit self-report data for males showed a greater anti-fat bias compared to females in the study by O'Brien et al., the implicit data failed to show a gender difference, and both males and females showed similar anti-fat bias. The authors speculated that the lower anti-fat bias for female participants evident in self-report measures may have been influenced by empathy toward the overweight, perhaps because societal weight bias reportedly has a greater negative impact on women. The current findings regarding greater anti-fat bias toward male compared to female targets are inconsistent with previous research, in which anti-fat bias was greater toward women targets (Hebl \& Turchin, 2005). However, this greater anti-fat bias toward women has not been consistently found in previous research (Puhl \& Brownell, 2006). The findings from the current study highlight the need for additional studies involving larger, more gender-balanced sample sizes. This would increase the possibility of obtaining representative data and thus facilitate generalizing from the data on perceptual bias.

Another hypothesis proposed at the outset of this study was that on the CPT, participants would demonstrate body-weight bias, with participants rating overweight individuals as less intelligent and less likely to gain employment than slim individuals. Data for the combined group from the CPT failed to provide support for the hypothesis; however, when data were analyzed for gender effects, it was revealed that men rated overweight individuals as less intelligent and less likely to gain employment. Thus, the second hypothesis was partially supported. The observed gender difference in this measure is consistent with previous research that found that male participants showed greater negativity toward overweight targets compared to normal weight targets (Brochu \& Morrison, 2007). The CPT also investigated if greater body-weight bias was attributed to 
one target gender more than the other. In other words, would body-weight bias be shown toward male targets but not toward female targets, or vice versa? The results from the CPT suggest that this is not the case; the gender of the person in the description did not have a significant effect on the rating scores. It should be noted that these data were analyzed controlling for influence of body weight of targets, to determine if the gender of the target by itself exerted an influence on perceived intelligence or job opportunities. Findings failed to show an effect for gender of target, suggesting that in the context of perceived intelligence and success, whether a person is slim or overweight could be more influential than the person's gender if the evaluators are male.

The fourth hypothesis in the current study compared findings on covert measures, explicit measures, and implicit measures, relative to intelligence and body-weight bias. Specifically, it was predicted that body-weight bias would be observed on the covert measure and the implicit measure, but not on the explicit measure, because explicit self-report measures are more vulnerable to social desirability effects. Scores on the AFA, which was the explicit self-report measure used, indicated a low anti-fat bias for male participants but not for female participants. When data were examined for correlations, although body-weight bias was observed on both the covert measure and the implicit measure, a correlation was not found between both measures. This may imply that these measures were not measuring the same variable and call into question the validity of the covert measure. However, it should be noted that the small group size may be relevant to explain the lack of significant correlations, and other tests similar to the CPT have been found to correlate with implicit measures (Brochu \& Morrison, 2007). Nevertheless, further research is required to clarify these matters. Correlation tests showed that results on the explicit measure were correlated with both the CPT and the IRAP. Specifically, participants who held negative views toward the overweight on the CPT scored higher on the AFA. Likewise, participants who scored higher on the AFA also showed increased implicit bias. This was not consistent with the hypothesis, as no relationship was expected between explicit and implicit measures because self-report measures are prone to social desirability effects (e.g., participants may not wish to explicitly agree with statements that are clearly negative toward obesity or overweight individuals). Furthermore, previous studies using both the AFA and the IRAP did not find correlations between implicit and explicit bias (Roddy et al., 2010). The inconsistency may be due to the focus on intelligence as the context of body-weight bias in the current study, whereas Roddy et al. (2010) examined more general positive-negative bias regarding body weight; however, this is a speculative explanation. Analysis of the data for subscales of the AFA revealed that Willpower was the highest scoring subscale on the test, and this was the only subscale that correlated with the scores on the IRAP. More data are required before a reasonable attempt can be made to interpret this finding.

A previously mentioned limitation of the current study may be the relatively small sample size, particularly because the number of female participants was less than the number of male participants. The smaller number of female participants could have exerted a confounding influence regarding the gender effects suggesting that males showed greater body-weight bias, but is unlikely to have been influential in the gender effects found in relation to the target stimuli (i.e., that greater body-weight bias was shown toward overweight men compared to overweight women). An additional problem was that the method used to measure current body weight of participants was also problematic, as the PIQ scale used did not have sufficient resolution to allow accurate analysis of the data. A direct measurement approach may have improved the accuracy of weight measures or BMI.

The current findings extend the growing literature on body-weight bias and, importantly, show that the bias may be more than generally unfavorable to the overweight - it appears that even perceptions of an individual's intelligence may be influenced by their body weight. The use of the relatively new behavioral measure, the 
IRAP, has allowed for an increased level of detail pertaining to the directionality and relativity of body-weight bias compared to the IAT and shows a pro-slim rather than antifat bias, in accordance with previous IRAP research literature (Roddy et al., 2010). Roddy et al. found a pro-slim bias in the context of positive-negative perceptions, and the current findings showed that this trend was also found in the context of intelligence, specifically, that perceptions of intelligence and success are related to the body weight of the person being evaluated. Indeed, all the three measures employed in the current study suggest a link between body weight and perceived intelligence. The current study was also informative regarding gender differences in body-weight bias and in relation to effects of gender of target stimuli. Male participants demonstrated increased levels of bias on all three measures in the study (covert, explicit, and implicit) and were found to be more biased when targets were men. These findings highlight the need for further research investigating gender effects in the context of body-weight bias. The study of implicit attitudes via a behavior analytic perspective has helped to provide a broadened understanding of the complexities involved in implicit attitudes, and challenged the dominance of what has been termed the associative meta-theoretical position in the field (Hughes, Barnes-Holmes, \& De Houwer, 2011). Further behavioral research into the type of relational responding involved in implicit attitudes may contribute to an extended body of knowledge.

\section{References}

AHERn, A. L., \& HETHERINGTON, M. M. (2006). The thin ideal and body image: An experimental study of implicit attitudes. Psychology of Addictive Behaviors, 20(3), 338-342.

BARNES-HOLMES, D., BARNES-HOLMES, Y., HAYDEN, E., MILNE, R., POWER, P., \& STEWART, I. (2006). Do you really know what you believe? Developing the Implicit Relational Assessment Procedure as a direct measure of implicit beliefs. The Irish Psychologist, 32, 169-177.

BARNES-HOLMES, D., BARNES-HOLMES, Y., STEWART, I., \& BOLES, S. (2010). A sketch of the Implicit Relational Assessment Procedure (IRAP) and the Relational Elaboration and Coherence (REC) model. The Psychological Record, 60, 527-542.

BARNES-HOLMES, D., MURPHY, A., BARNES-HOLMES, Y., \& STEWART, I. (2010). The Implicit Relational Assessment Procedure (IRAP): Exploring the impact of private versus public contexts and the response latency criterion on pro-White and anti-Black stereotyping among White Irish individuals. The Psychological Record, 60, 57-66.

BARNES-HOLMES, D., MURTAGH, L., BARNES-HOLMES, Y., \& STEWART, I. (2010). Using the Implicit Association Test and the Implicit Relational Assessment Procedure to measure attitudes toward meat and vegetables in vegetarians and meat-eaters. The Psychological Record, 60, 287-306.

BARNES-HOLMES, D., WALDRON, D., BARNES-HOLMES, Y., \& STEWART, I. (2009). Testing the validity of the Implicit Relational Assessment Procedure (IRAP) and the Implicit Association Test (IAT): Measuring attitudes towards Dublin and country life in Ireland. The Psychological Record, 59, 389-406.

BROCHU, P. M., \& MORRISON, M. A. (2007). Implicit and explicit prejudice toward overweight and average weight men and women: Testing their correspondence and relation to behavioural intentions. Journal of Social Psychology, 147, 681-706. doi:10.3200/SOCP.147.6.681-706.

CATERSON, I. D., \& GILL, T. P. (2002). Obesity: Epidemiology and possible prevention. Best Practice \& Research: Clinical Endocrinology and Metabolism, 16, 595-610. doi:10.1053/beem.2002.0228 
CRANDALL, C. S. (1994). Prejudice against fat people: Ideology and self-interest. Journal of Personality \& Social Psychology, 66, 882-894.

DE HOUWER, J. (2002). The Implicit Association Test as a tool for studying dysfunctional associations in psychopathology: Strengths and limitations. Journal of Behaviour Therapy \& Experimental Psychiatry, 33, 115-133.

DOVIDIO, J. F., \& FAZIO, R. H. (1992). New technologies for the direct and indirect assessment of attitudes. In J. Tanur (Ed.), Questions about survey questions: Meaning, memory, attitudes, and social interaction (pp. 204-237). New York, NY: Russell Sage Foundation.

FREIDMAN, K. E., REICHMANN, S. K., COSTANZO, P. R., ZELLI, A., ASHMORE, J. A., \& MUSTANTE, G. J. (2005). Weight stigmatization and ideological beliefs: Relation to psychological functioning in obese adults. Obesity Reviews, 13, 907-916.

GAPINSKI, K. D., SCHWARTZ, M. B., \& BROWNELL, K. D. (2006). Can television change anti-fat attitudes and behaviour? Journal of Applied BioBehavioural Research, 11, 1-28. doi:10.1111/j.1751-9861.2006.tb00017.x

GREENWALD, A. G., MCGHEE, A. D., \& SCHWARTZ, J. L. (1998). Measuring individual differences in implicit cognition: The Implicit Association Test. Journal of Personality \& Social Psychology, 74, 1464-1480. doi:10.1037/0022-3514.74.6.1464

GREENWALD, A. G., NOSEK, B. A., \& BANAJI, M. R. (2003). Understanding and using the Implicit Association Test: An improved scoring algorithm. Journal of Personality \& Social Psychology, 85, 198-216. doi:10.1037/0022-3514.85.2.197

HEBL, M. R., \& TURCHIN, J. M. (2005). The stigma of obesity: What about men? Basic and Applied Social Psychology, 3, 267-275. doi:10.1207/s15324834basp2703_8

HUGHES, S., BARNES-HOLMES, D., \& DE HOUWER, J. (2011). The dominance of associative theorizing in implicit attitude research: Propositional and behavioral alternatives. The Psychological Record, 61, 465-498.

JAMES, P. T., LEACH, R., KALAMARA, E., \& SHAYEGHI, M. (2001). The worldwide obesity epidempic. Obesity Research, 9, 228-233. doi:10.1038/oby.2001.123

LEWIS, R. J., CASH, T. F., JACOBI, L., \& BUBB-LEWIS, C. (1997). Prejudice toward fat people: The development and validation of the anti-fat attitudes test. Obesity Research, 5, 297-307. doi:10.1002/j.1550-8528.1997.tb00555.X

MCKENNA, I., BARNES-HOLMES, D., BARNES-HOLMES, Y., \& STEWART, I. (2007). Testing the fake-ability of the Implicit Relational Assessment Procedure (IRAP): The first study. International Journal of Psychology \& Psychological Therapy, 7, 253-268.

MOKDAD, A. H., FORD, E. S., BOWMAN, B. A., DIETZ, W. H., VINICOR, F., BALES, V. S., \& MARKS, J. S. (2003). Prevalence of obesity, diabetes, and obesity-related health risk factors. Journal of the American Medical Association, 289, 76-79. doi:10.1001/jama.289.1.76

O’BRIEN, K. S., HUNTER, J. A., \& BANKS, M. (2006). Implicit anti-fat bias in physical educators: Physical attributes, ideology, and socialization. International Journal of Obesity, 1, 308-314. doi:10.1038/sj.ijo.0803398

O’BRIEN, K. S., HUNTER, J. A., HALBERSTAT, J., \& ANDERSON, J. (2007). Body image and explicit and implicit anti-fat attitudes: Physical attributes, ideology, and socialization. Body Image, 4(3), 249-256. doi:10.1016/j.bodyim.2007.06.001

O’BRIEN, K. S., LATNER, J. A., HALBERSTAT, J. , HUNTER, J. A., ANDERSON, J., \& CAPUTI, P. (2008). Do antifat attitudes predict antifat behaviors? Obesity, 16, S87-S92.

PUHL, R. M., \& BROWNELL, K. (2006). Confronting and coping with weight stigma: An investigation of overweight and obese adults. Obesity, 14, 1802-1815. 
RODDY, S., STEWART, I., \& BARNES-HOLMES, D. (2010). Anti-fat, pro-slim, or both? Using two reaction time based measures to assess implicit attitudes to the slim and overweight. Journal of Health Psychology, 15, 416-425. doi:10.1177/1359105309350232

SCHWARTZ, M. B., VARTANIAN, L. R., NOSEK, B. A., \& BROWNELL, K. D. (2006). The influence of one's own body weight on implicit and explicit anti-fat bias. Obesity, 14, 4404-4447.

TEACHMAN, B. A., \& BROWNELL, K. D. (2001). Implicit anti-fat bias among health professionals: Is anyone immune? International Journal of Obesity, 25, 1525-1531.

TEACHMAN, B. A., GAPINSKI, K. D., BROWNELL, K. D., RAWLINS, M., \& YEYARAM, S. (2003). Demonstrations of implicit anti-fat bias. Health Psychology, 22, 68-78.

WORLD HEAlth ORGANIZATION (WHO). (2000). Obesity: Preventing and managing the global epidemic (WHO Obesity Technical Report Series No. 894). Geneva, Switzerland: Author. 


\section{Appendix A}

\section{Covert Perception Task: Written Stimuli and Questionnaire}

\section{Condition 1: Male Overweight}

Gerry is a 22-year-old man. He is $5 \mathrm{ft} 8$ in $(173 \mathrm{~cm})$ tall, weighs $\mathbf{2 5 0} \mathbf{~ l b ~ ( 1 1 3 ~} \mathbf{~ k g})$, has dark hair, and brown eyes. Gerry works part-time in a busy fast-food restaurant. He is currently in his first year of an arts degree in NUI Maynooth. He enjoys socialising with friends and goes for nights out on most weekends. Gerry's friends describe him as generally happy and easy going. In time Gerry would like to teach in a secondary school.

\section{Condition 2: Male Normal Weight}

Gerry is a 22-year-old man. He is $5 \mathrm{ft} 8$ in $(173 \mathrm{~cm})$ tall, weighs $150 \mathbf{~ l b ~ ( 6 8 ~} \mathbf{~ k g})$, has dark hair, and brown eyes. Gerry works part-time in a busy fast-food restaurant. He is currently in his first year of an arts degree in NUI Maynooth. He enjoys socialising with friends and goes for nights out on most weekends. Gerry's friends describe him as generally happy and easy going. In time Gerry would like to teach in a secondary school.

\section{Condition 3: Female Overweight}

Geraldine is a 22-year-old woman. She is $5 \mathrm{ft} 8$ in $(173 \mathrm{~cm})$ tall, weighs $250 \mathbf{~ l b ~ ( 1 1 3 ~} \mathbf{~ k g})$, has dark hair, and brown eyes. Geraldine works part-time in a busy fast-food restaurant. She is currently in her first year of an arts degree in NUI Maynooth. She enjoys socialising with friends and goes for nights out on most weekends. Geraldine's friends describe her as generally happy and easy going. In time Geraldine would like to teach in a secondary school.

\section{Condition 4: Female Normal Weight}

Geraldine is a 22-year-old woman. She is $5 \mathrm{ft} 8$ in $(173 \mathrm{~cm})$ tall, weighs $150 \mathbf{~ l b ~}(\mathbf{6 8} \mathbf{~ k g})$, has dark hair, and brown eyes. Geraldine works part-time in a busy-fast food restaurant. She is currently in her first year of an arts degree in NUI Maynooth. She enjoys socialising with friends and goes for nights out on most weekends. Geraldine's friends describe her as generally happy and easy going. In time Geraldine would like to teach in a secondary school.

\section{Five-Item Questionnaire}

- The person in the description is intelligent.

- The person in the description is hard working.

- The person has a good chance of becoming a teacher.

- The person spends a lot of time studying.

- If I were an employer, I would hire the person. 
Appendix B

\section{Participant Information Questionnaire}

The screenshots below show each screen of the Participant Information Questionnaire as presented to the participants.

\section{Please answer the following questions about yourself}

Age

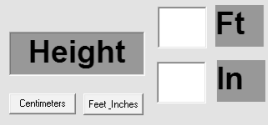

\section{Please Click Here to Continue}

Figure B.1. Age and height.

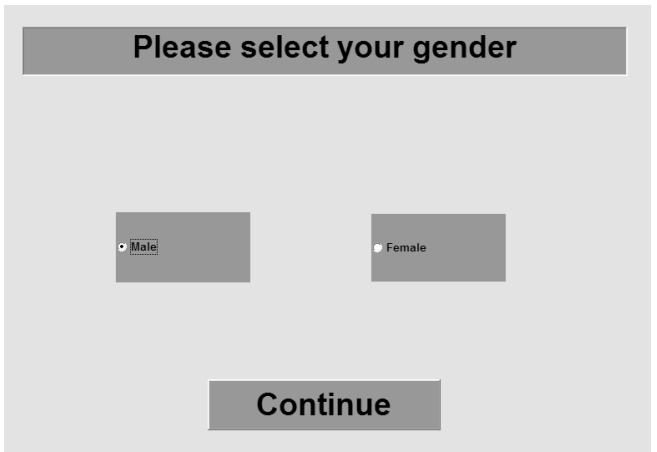

Figure B.2. Gender.

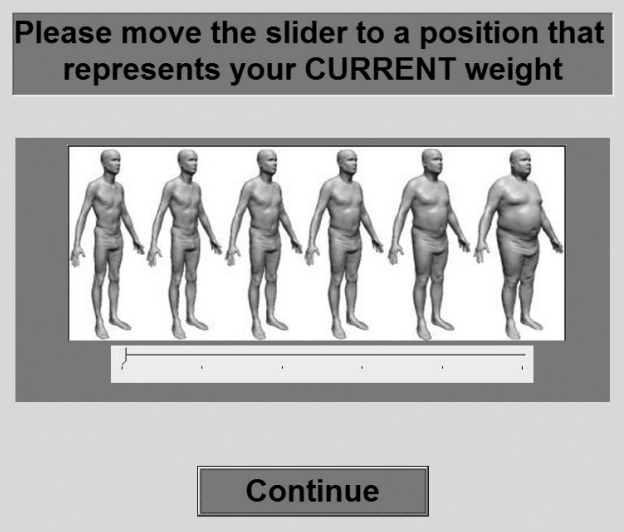

Figure B.3. Male current BMI. 


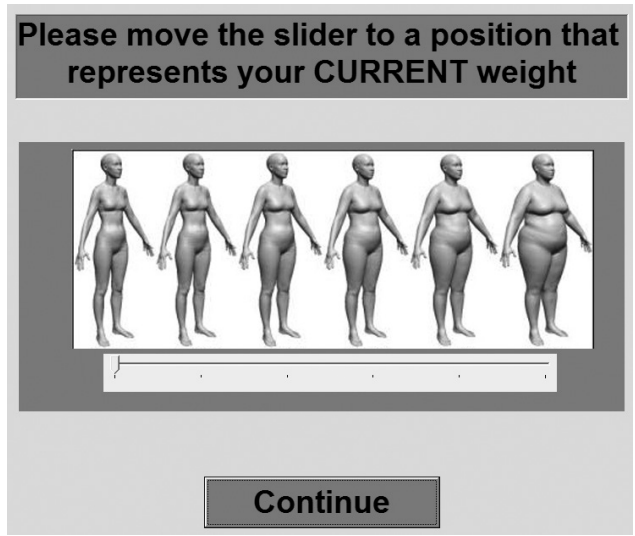

Figure B.4. Female current BMI.

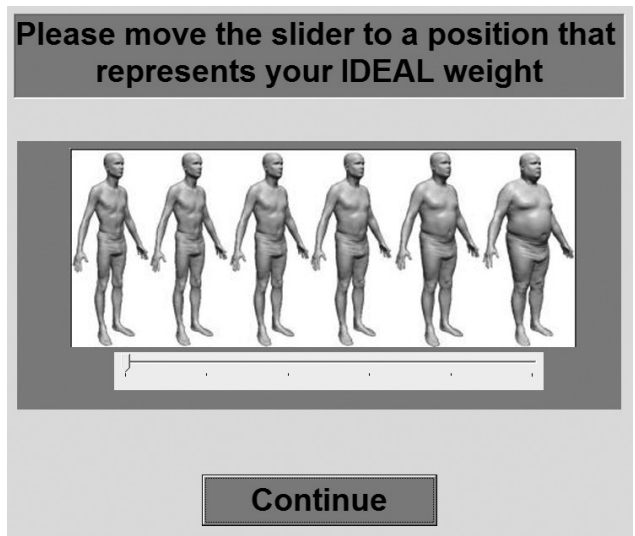

Figure B.5. Male ideal BMI.

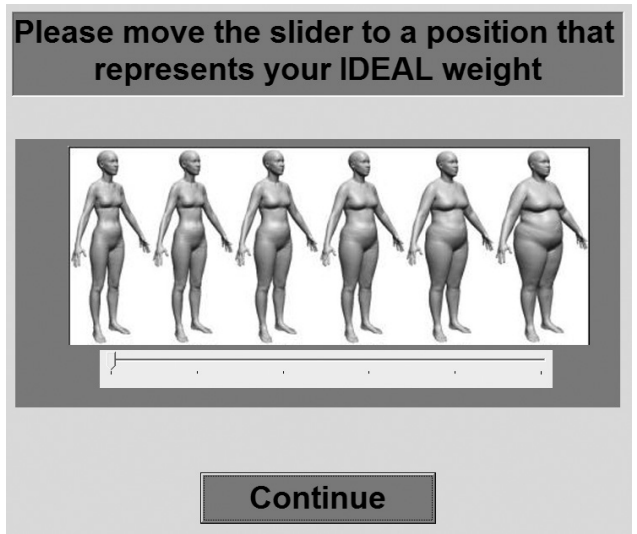

Figure B.6. Female ideal BMI. 
Copyright of Psychological Record is the property of Psychological Record and its content may not be copied or emailed to multiple sites or posted to a listserv without the copyright holder's express written permission. However, users may print, download, or email articles for individual use. 